\title{
Absolute yields of the exciton induced desorption at the surface of a solid rare gases
}

\author{
I. Arakawa and T. Adachi \\ Department of Physics, Gakushuin University, Mejiro, Tokyo 171-8588, Japan \\ E-mail: ich.arakawa@gakushuin.ac.jp \\ T. Hirayama \\ Department of Physics, Rikkyo University, Ikebukuro, Tokyo 171-8501, Japan \\ M. Sakurai \\ Department of Physics, Kobe University, Nada, Kobe 657-8501, Japan
}

Received 10 December, 2002

\begin{abstract}
Absolute yields of the photo-induced desorption at the surface of solid rare gases were studied in the excitonic excitation region. Both metastable and total desorption yields depend strongly on excitation energy and film thickness of rare gas solids. The absolute desorption yields and their dependence on film thickness were quantitatively reproduced by a simulation based on the diffusion of excitons in the bulk and the kinetic energy release by a cavity ejection mechanism and an excimer dissociation one followed by internal sputtering.
\end{abstract}

PACS: 71.35.-y, 79.20.La

\section{Introduction}

When the surface of a solid rare gas is irradiated with vacuum ultraviolet light, it can eject atoms and clusters in ground, electronically and vibrationally excited, or ionized states. A variety of desorption mechanisms have been proposed for each desorbed species and for the excitation energy region. Two types of mechanisms are known to play an essential role in the desorption induced by the excitonic excitation; a cavity ejection (CE) mechanism is due to the repulsive interaction between the excited atom with an inflated electron cloud and the matrix with a negative electron affinity and an excimer dissociation (ED) one is the dissociation of an excimer in the vicinity of the surface. The investigation of the desorption phenomena initiated by the excitonic excitation is one of the most powerful tools to reveal the dynamic characters of the exciton in rare gas solids [1]. The desorption of excited neutral particles of $\mathrm{Ne}$ and $\mathrm{Ar}$ has extensively been investigated for two decades [2]; the close relationship between exciton formation [3] and the detail of the desorption mechanisms [4] has been almost fully elucidated. On the other hand, the desorption mechanism of the ground state neutral, as well as its desorption yield, has not been yet clarified though it can safely be said that they are the main component in the desorbed species.

We report the measured results on absolute yields of the metastable desorption and the total one which are induced by the excitonic excitation from the surfaces of solid Ne, Ar, and Kr. We also show that the experimental results for $\mathrm{Ne}$ and $\mathrm{Kr}$ are satisfactorily reproduced by the simple model simulation of the desorption process.

\section{Desorption mechanism}

The negative electron affinity of the matrix is known to be essential for the CE process to have a repulsive interaction between the excited atom and the surrounding ground state atoms. The metastables desorbed through the CE process were observed for the solid whose electron affinity $E_{a}$ is negative, namely, solid $\mathrm{Ne}\left(E_{A}=-1.4 \mathrm{eV}\right)$ and $\mathrm{Ar}(-0.4 \mathrm{eV})$, but not for solid $\mathrm{Kr}(0.3 \mathrm{eV})$ and $\mathrm{Xe}(0.5 \mathrm{eV})$ [5]. The kinetic energy $E_{k}$ of the metastable desorbed through 
the $\mathrm{CE}$ process is originated from the lattice distortion energy around the exciton, which can roughly be estimated from the difference between the excitation energy $E_{x}$ of the exciton and the excitation energy $E_{g}$ of the corresponding state of an isolated atom in the gas phase [1]. The energy difference, $E_{x}-E_{g}$, is divided among three terms, $E_{k}$ : the kinetic energy of a desorbing particle, $E_{\mathrm{coh}}$ : the cohesive energy of an atom on the surface, and $E_{\text {latt }}$ : the energy absorbed in the lattice [6]. It has been revealed that $30-70 \%$ of the lattice distortion energy is transferred into the kinetic energy of the desorbing metastable from the surface exciton $\mathrm{S} 1$ of $\mathrm{Ne}$ and $\mathrm{Ar}$ [4]. It is found also experimentally studied by the molecular dynamic calculation [7]. In the case of $\mathrm{Ne}$, for example, $E_{k}$ of the metastable which is desorbed by the first order surface exciton is $0.18 \mathrm{eV}$ at the peak of the distribution, while the excitation energy $E_{x}$ is $(17.17 \pm 0.03) \mathrm{eV}$ and $E_{g}$ of the corresponding $2 p^{5} 3 \mathrm{~S}$ states are 16.619-16.848 eV [8]. The excitation energy $E_{x}$ of the bulk exciton $\mathrm{B} 1$ of $\mathrm{Ne}$ is $(17.57 \pm 0.03) \mathrm{eV}$. Considering the low cohesion energy, $0.019 \mathrm{eV}$, of $\mathrm{Ne}$, the lattice distortion energy, $E_{x}-E_{g}$, of $0.9-0.7 \mathrm{eV}$ is large enough to squeeze the excited atom out of a second or third underlying layer to vacuum. The squeezed excited atom may push overlying atoms forward. This internal sputtering process can result in a large total desorption yield of Ne. In solid Ar, though the lattice distortion energy is estimated at about $0.7-0.4 \mathrm{eV}$, the internal sputtering process must be less efficient because of its larger cohesion energy of $0.068 \mathrm{eV}$.

Dissociative relaxation of a rare gas excimer in the gas phase yields the kinetic energy of about $1 \mathrm{eV}$ [9]. It is known that the ED process in solid produces crystal defects [10]. If ED occurs in the surface vicinity of a rare gas solid, a large number of atoms in the surface layer will be released into vacuum by the internal sputtering process.

\section{Experimental}

The experimental apparatus for the photostimulated desorption (PSD) study was settled at BL5B beam line in the UVSOR facilities of the Institute for Molecular Science, Okazaki, Japan [11-13]. A solid rare gas film was prepared on a platinum substrate at a temperature of about $6 \mathrm{~K}$ which was attached to a liquid helium cryostat in an ultra-high vacuum chamber with a base pressure below $10^{-8} \mathrm{~Pa}$. The amount of the gas condensed on the substrate, the film thickness, was calculated from the exposure, which was the product of pressure and the duration of deposition, assuming the condensation coefficient to be unity.
The wavelength resolution, $\lambda / \Delta \lambda$, of the vacuum ultraviolet light source was about $500 \pm 200$ in the range of $\lambda$ between 20 and $100 \mathrm{~nm}$. The light intensity was monitored by the photoelectron current from a gold plated mesh, through which the light beam was introduced onto the sample surface. We adopted the value of 0.07 electrons/photon as the photoelectron yield at the gold surface, which is the average value of the published data by Samson [14] between 52 and $100 \mathrm{~nm}$ of wavelength. The intensity of photon flux ranged between $10^{11}$ and $10^{12}$ photons / s in continuous mode and between $10^{5}$ and $10^{6}$ photons/pulse in pulse mode within a diameter of $3 \mathrm{~mm}$ at the sample.

\subsection{Metastable desorption measurement}

The monochromatized photon beam is pulsed by a mechanical chopper in order to measure the time-of-flight (TOF) spectra of the desorbed metastable atoms. The width and the interval of the pulsed beam are $15 \mu$ s and $2.5 \mathrm{~ms}$, respectively. The photon beam is incident at $20 \mathrm{deg}$ of the normal direction of the sample surface.

The desorbed metastable atoms are detected by an open electron multiplier tube (EMT, Hamamatsu, R595) with a $\mathrm{CuBe}$ dynode as a first electrode. The EMT is fixed at a distance of $360 \mathrm{~mm}$ from the sample in the normal direction of the sample. The diameter of the entrance of EMT is $8 \mathrm{~mm}$, which corresponds to the detection solid angle of $3.1 \cdot 10^{-5}$ sr. The lifetimes of the metastable $\left({ }^{3} P_{0,2}\right)$ rare gas atoms are known to be much longer than the flight time of the detected atoms which is less than $1 \mathrm{~ms}$ in our experimental system. The charged particles are rejected by applying suitable voltages to the grid mesh in front of the detector and the 1st dynode of EMT. In order to calculate the overall metastable yields, which were integrated for all direction in half space, we utilized the angular distribution of the desorbed metastables which have already been reported by our group [15-17].

\subsection{Total desorption measurement}

The total desorption rate was calculated from the pumping speed and the rise of the partial pressure in the vacuum chamber during irradiation of the sample. The pumping speed of the turbo molecular pump and cold surfaces for desorbed rare gas was determined from the pressure measured by an extractor gauge installed in the chamber and from the flow rate calibrated volumetrically using a reference volume and a Baratron pressure gauge as a reference. A little rise of the partial pressure during irradiation was detected by a quadrupole mass spectrometer which was calibrated against the extractor gauge. It should be noted that 
the uncertainty of the relative sensitivity of the extractor gauge was cancelled in the present method of determining the desorption rate.

\subsection{Accuracy of measurements}

In the metastable yield determination, the uncertainty of the results was estimated to be $\pm 30 \%$ by a quadrature sum of all sources of error; light intensity measurement, detection efficiency of the photomultiplier, geometrical configuration, etc. The main part of the error came from the estimation of the amount of higher order light from the monochromator [12,18].

The uncertainty in determining the absolute yield of the total desorption was estimated as large as $\pm 1 / 3$ of the order of magnitude; an error bar ranging from $-50 \%$ to $+100 \%$ of the obtained value should be added to the obtained values. In addition to the uncertainty in the intensity measurement of the incident photon, it was caused by difficulties in determining the sensitivity of the mass spectrometer and the pumping speed for the desorbed species.

Noticeable changes in the desorption yields were observed after a few hours exposure of the sample in the chamber at a pressure of lower $10^{-8} \mathrm{~Pa}$ range, where the main component was a rare gas itself. It was probably due to the adsorption of the common residual gases, $\mathrm{H}_{2}, \mathrm{H}_{2} \mathrm{O}, \mathrm{CO}$, in the UHV system $[19,20]$. The uncertainty caused by this impurity effect is within the above mentioned error bar in the present experiment.

\section{Desorption of $\mathrm{Ne}$}

\subsection{Experimental results}

The desorption yields of $\mathrm{Ne}$ metastable in the excitonic excitation region is shown in Fig. 1 as a

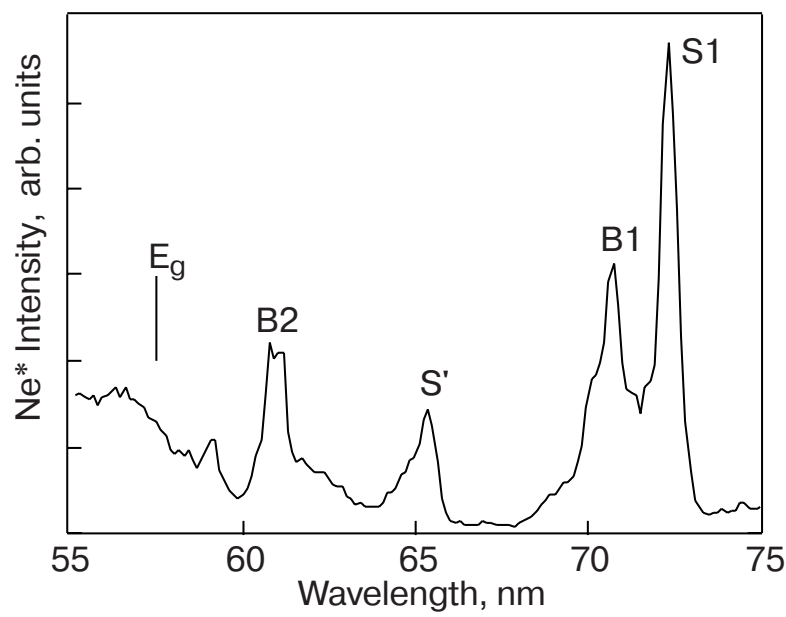

Fig. 1.The desorption yields of the Ne metastable in the excitonic excitation region as a function of incident wavelength.

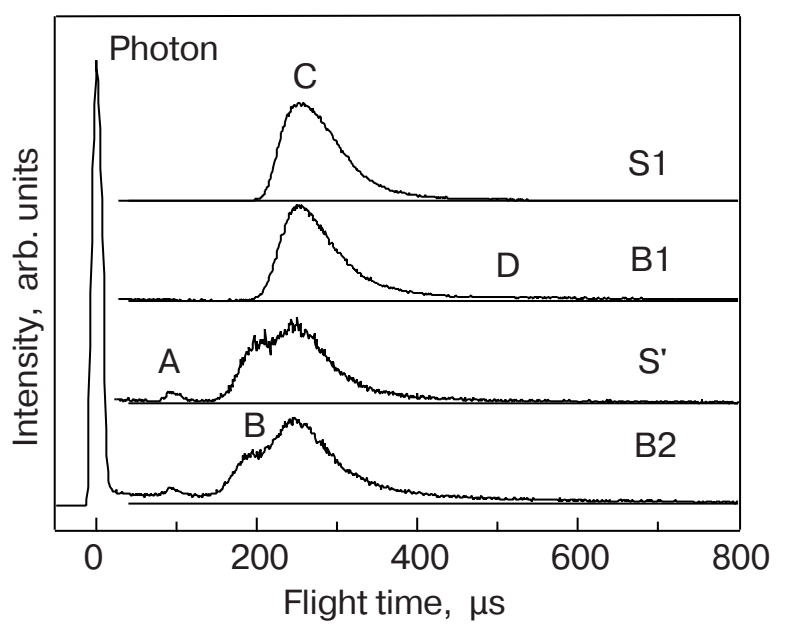

Fig. 2. The time-of-flight spectra of the metastable Ne desorbed by the excitation of the four types of excitons.

function of incident wavelength. Most of the observed peaks in the figure can be assigned to a series of the bulk (B) and the surface (S) excitons reported by Saile and Koch [21], where $S^{\prime}$ peak is caused by the exciton in the $2 p^{5} 3 p$ state which is allowed at the surface because of the reduced symmetry [22]. The four TOF spectra of the Ne metastable in Fig. 2 were obtained by the excitations at S1, B1, S', and B2 excitons.

The wavelength dependence of the absolute yield of the total photo-desorption in the range between 52 and $77 \mathrm{~nm}$ is shown in Fig. 3 for a film thickness of 73 atomic layers, where the peaks caused by the exciton excitation are also clearly seen. The absolute value of the desorption yield in the figure represents the average number of the Ne atoms desorbed by one incident photon. The large and continuous background was due to the desorption induced by ionization by the second

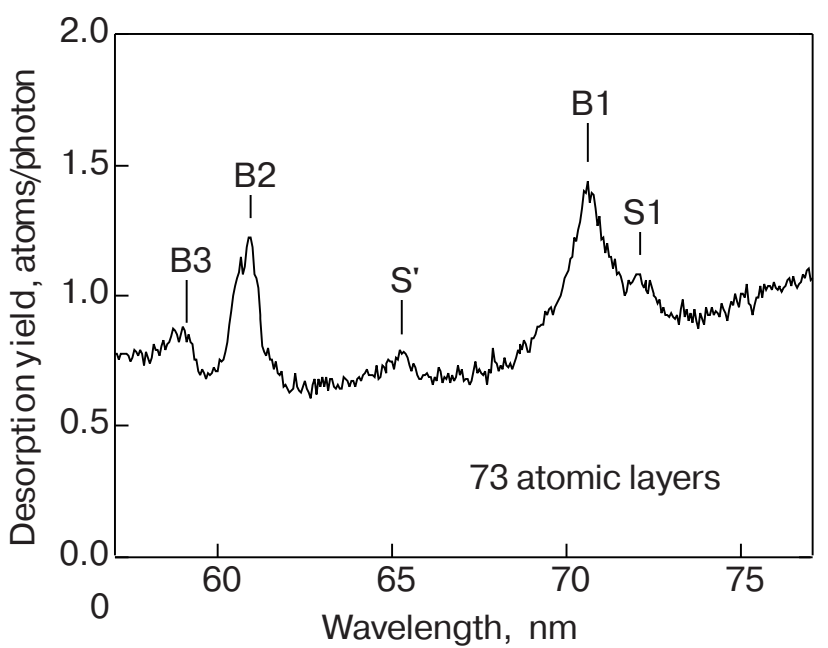

Fig. 3. The absolute yields of total desorption of the Ne in the excitonic excitation region as a function of incident wavelength. 
The absolute yields of the metastable and the total desorption from solid $\mathrm{Ne}$, Ar, and $\mathrm{Kr}$

\begin{tabular}{|c|c|c|c|c|}
\hline Rare gases & $\begin{array}{c}\begin{array}{c}\text { Initial } \\
\text { excitation }\end{array} \\
\end{array}$ & Desorbed species & Yield, atoms / photon & Desorption mechanisms \\
\hline \multirow{8}{*}{$\mathrm{Ne}$} & \multirow{2}{*}{$\mathrm{S} 1$} & metastable & $(2.3 \pm 0.7) \cdot 10^{-3}$ & $\mathrm{CE}$ \\
\hline & & total & 0.3 & $\mathrm{CE}+\mathrm{S} / \mathrm{CE}(? \%)+\mathrm{S} / \mathrm{ED}(? \%)$ \\
\hline & \multirow{2}{*}{$\mathrm{S}^{\prime}$} & metastable & $(7.8 \pm 2.3) \cdot 10^{-4}$ & $\mathrm{CE}+\mathrm{ED}(\leq 1 \%)$ \\
\hline & & total & 0.1 & $\mathrm{CE}+\mathrm{S} / \mathrm{CE}(? \%)+\mathrm{S} / \mathrm{ED}(? \%)$ \\
\hline & \multirow{2}{*}{ B1 } & metastable & $(1.4 \pm 0.4) \cdot 10^{-3}$ & $\mathrm{CE}+\mathrm{S} / \mathrm{CE}(\mathrm{a}$ few $\%)$ \\
\hline & & total & $1.6 \pm 0.3$ & $\mathrm{~S} / \mathrm{CE}+\mathrm{CE}(\approx 10 \%)+\mathrm{S} / \mathrm{ED}(\mathrm{a}$ few $\%)$ \\
\hline & \multirow{2}{*}{ B2 } & metastable & $\approx 1 \cdot 10^{-3}$ & $\mathrm{CE}+\mathrm{S} / \mathrm{CE}(\mathrm{a}$ few $\%)+\mathrm{ED}(\leq 1 \%)$ \\
\hline & & total & $1 \pm 0.2$ & $\mathrm{~S} / \mathrm{CE}+\mathrm{CE}(\approx 10 \%)+\mathrm{S} / \mathrm{ED}($ a few $\%)$ \\
\hline \multirow{8}{*}{$\mathrm{Ar}$} & \multirow{2}{*}{ S1 } & metastable & $\approx 1 \cdot 10^{-5}$ & $\mathrm{CE}$ \\
\hline & & total & 0.1 & $\mathrm{CE}$ \\
\hline & \multirow{2}{*}{$\mathrm{S} 2$} & metastable & not determined & $\mathrm{CE}+\mathrm{ED}$ \\
\hline & & total & 0.1 & $\mathrm{CE}$ \\
\hline & \multirow{2}{*}{ B1 } & metastable & not determined & $\mathrm{CE}$ \\
\hline & & total & 0.23 & $\mathrm{CE}+\mathrm{S} / \mathrm{ED}$ \\
\hline & \multirow{2}{*}{ B2 } & metastable & not determined & $\mathrm{CE}+\mathrm{ED}$ \\
\hline & & total & 0.16 & $\mathrm{CE}+\mathrm{S} / \mathrm{ED}$ \\
\hline \multirow{8}{*}{$\mathrm{Kr}$} & $\mathrm{S} 1(3 / 2)$ & total & 0.015 & $\mathrm{~S} / \mathrm{ED}$ \\
\hline & $\mathrm{S} 1(1 / 2)$ & total & 0.01 & $\mathrm{~S} / \mathrm{ED}$ \\
\hline & \multirow{2}{*}{$\mathrm{S} 2(3 / 2)$} & metastable & not determined & ED \\
\hline & & total & $\leq 10^{-3}$ & $\mathrm{~S} / \mathrm{ED}$ \\
\hline & $\mathrm{B} 1(3 / 2)$ & total & 0.03 & $\mathrm{~S} / \mathrm{ED}$ \\
\hline & $\mathrm{B} 1(1 / 2)$ & total & 0.02 & $\mathrm{~S} / \mathrm{ED}$ \\
\hline & \multirow{2}{*}{$\mathrm{B} 2(3 / 2)$} & metastable & not determined & ED \\
\hline & & total & $\leq 10^{-3}$ & $\mathrm{~S} / \mathrm{ED}$ \\
\hline
\end{tabular}

or higher order light from the monochromator. The peaks caused by the bulk excitons, B1 and B2, develop at the film thickness of a few tens atomic layers and the absolute yields seem to be saturated at around 1.6 and 1 atoms/photon, respectively, for the film thicker than 100 atomic layers. The peak heights due to the surface excitons seem to keep constant values of about 0.3 and 0.1 atoms / photon for S1 and $\mathrm{S}^{\prime}$, respectively, over the entire thickness range as expected for the surface excitations. The absolute yields of the metastable and the total desorption by the four different excitonic excitations are summarized in Table.

\subsection{Schematic model of the deSorption proceSS}

The mechanism which works in each desorption process and the branching ratio of the relaxation cascade which leads to the desorption can be deduced from a careful examination of the TOF spectra of the metastables and the absolute yields of the metastable and the total desorption. The mechanisms which work in the desorption process are also listed in Table.
The higher kinetic energy peak $A\left(E_{k}=(1.4 \pm\right.$ $\pm 0.1) \mathrm{eV}$ ) [23] in Fig. 2, which is only observed by the excitation of the higher order excitons ( $\mathrm{S}^{\prime}$ and B2), is caused by the excimer dissociation of the highly excited dimer: $\mathrm{Ne}_{2}^{* *} \rightarrow \mathrm{Ne}+\mathrm{Ne}^{*}+E_{k}$. This is also the case for Ar [24]. The lower kinetic energy peak, that is $\mathrm{CE}$ one, is obviously composed of two components $\mathrm{B}\left(E_{k}=(0.21 \pm 0.02) \mathrm{eV}\right)$ and $\mathrm{C}\left(E_{k}=\right.$ $=(0.18 \pm 0.02) \mathrm{eV})$ [12]. The metastables which compose the peaks $\mathrm{B}$ and $\mathrm{C}$ are ejected directly from the surface excitons $\mathrm{S}^{\prime}$ and $\mathrm{S} 1$, respectively [15]. The difference in the kinetic energy is caused by the difference in the magnitude of the repulsive interaction. The low energy tail $D$ is only observed by the bulk excitations (B1 and B2) [15] and is likely caused by the bulk exciton trapped in the underlying layer just below the surface. They are ejected into the vacuum, loosing their kinetic energy by collision with atoms in the overlayer. These TOF spectra can be used as a fingerprint to identify each exciton. These spectra also show clearly the relaxation channel of the excitons 
from the higher energy state to the lower one; from B1 to $\mathrm{S} 1$, from $\mathrm{S}^{\prime}$ to $\mathrm{S} 1$ and excimer, and from $\mathrm{B} 2$ to $S^{\prime}$, $\mathrm{S} 1$, and excimer.

The quantum efficiency of the exciton creation can be estimated from the photo-absorption coefficient of solid Ne at the wavelength corresponding to the bulk exciton (B1) excitation. We assume here that the excitation probability of $\mathrm{S} 1$ is the same as that of B1 in each layer at their own excitation energy. The estimation based on the data by Pudewill et al. [25] leads to the value of 0.1 excitons/photon in each layer.

The relaxation cascade of the first order surface exciton S1 of solid $\mathrm{Ne}$ is schematically shown in Fig. 4. Incident light of the wavelength of $72.2 \mathrm{~nm}$ will excite surface excitons with the probability of 0.1 excitons / photon in the first layer. 90\% of the incident photons pass through the solid Ne film and will be scattered or absorbed at the substrate. The surface exciton may decay radiatively or may be desorbed by the $\mathrm{CE}$ mechanism as a metastable in ${ }^{3} P_{2,0}$ state or as an excited atom in ${ }^{3} P_{1}$ or ${ }^{1} P_{1}$ state which decay into the ground state with a short lifetime of 1-10 ns. In our study, we have determined the absolute yields of the metastable desorption, $0.0023 \mathrm{Ne}^{*} /$ photon, and of the total desorption, $0.3 \mathrm{Ne} /$ photon. Considering the absolute total desorption yield at S1 excitation of 0.3 atoms/photon, the value, 0.1 excitons / photon, of the initial excitation probability must be underestimated since it is likely that a surface exciton can be desorbed only a single excited atom in the CE process. The larger desorption yield may be explained by other desorption processes: the desorption of a dimer by the $\mathrm{CE}$ process and the ED process which yields two or more desorbing atoms. However, the branching ratio to these two processes must be far smaller than that to the single atom desorption by the CE process. Another possibility is the excitation by the light reflected at the substrate surface. If this is the case, the initial excitation probability doubles at most. In any case, it can be concluded that almost all the surface excitons yield the desorption of one Ne atom or, in other words, that the desorption probability of the surface excitons S1 is almost unity. The comparison between the metastable desorption yield and the total one shows that about $1 \%$ of the desorbed $\mathrm{Ne}$ atoms are in the metastable state.

The above-mentioned scheme cannot be applied to the analysis of the desorption yield caused by $\mathrm{S}^{\prime}$ excitation because the quantum efficiency for $\mathrm{S}^{\prime}$ creation can not be estimated in the same manner; the excitation of $2 p^{5} 3 p$ state is only allowed at the surface of the solid. It can be said from the comparison between the total desorption yields by $\mathrm{S}^{\prime}$ and by $\mathrm{S} 1$, if we assume that the desorption probability of $\mathrm{S}^{\prime}$ exciton is also unity, that the excitation cross section of $S^{\prime}$ is one third of that of S1. The metastable TOF spectrum by $S^{\prime}$ excitation shows that almost two thirds of $S^{\prime}$ exciton decays into $\mathrm{S} 1$ state at the surface before the desorption and that a few $\%$ of $\mathrm{S}^{\prime}$ forms excimers in a higher excited state. It is also worth noting that the ratio of the number of the metastable species to the to-

photon $72.2 \mathrm{~nm}$

radiative decay

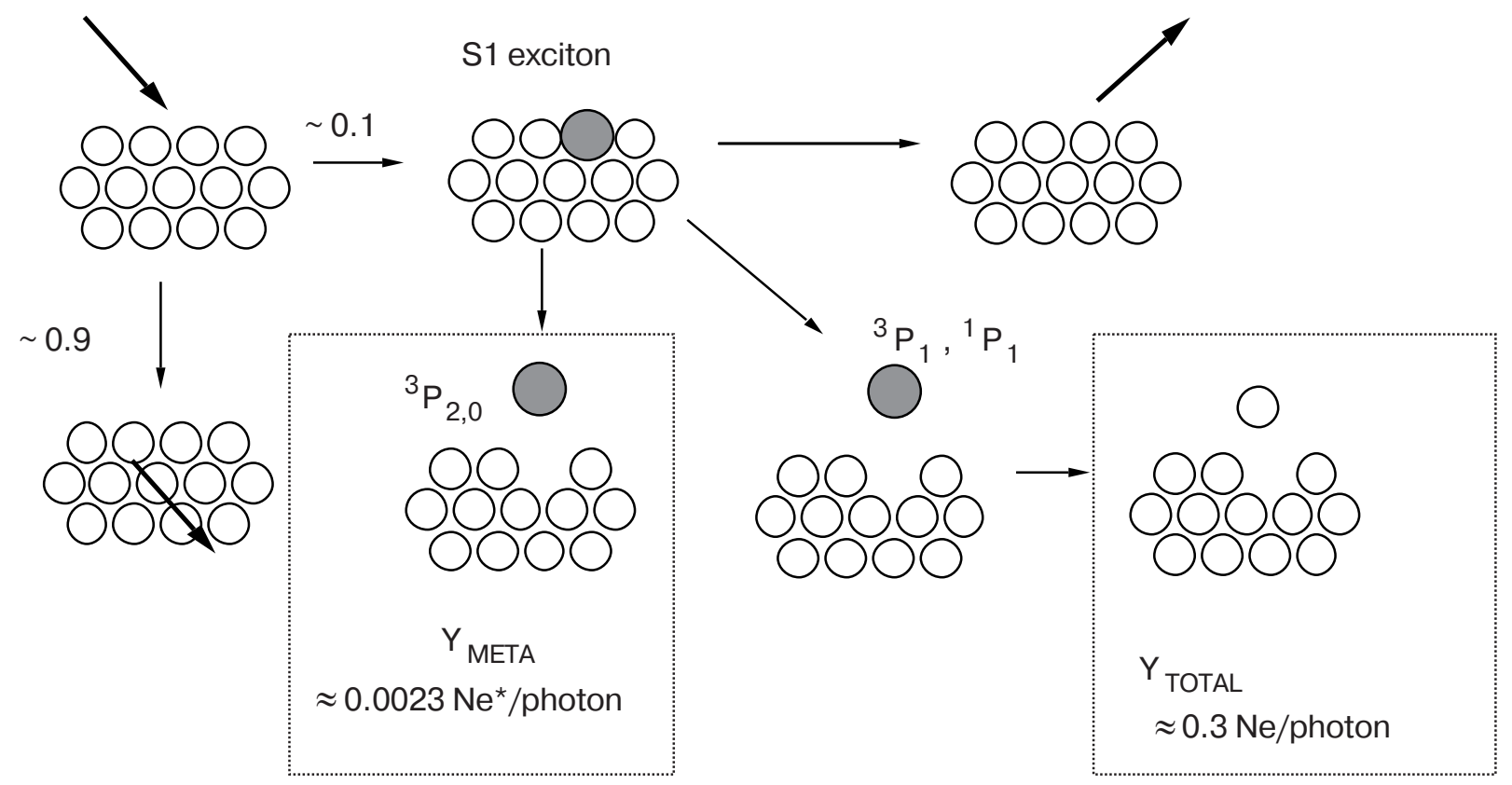

Fig. 4. Schematic chart of the relaxation cascade of the first order surface exciton S1 in solid Ne. 


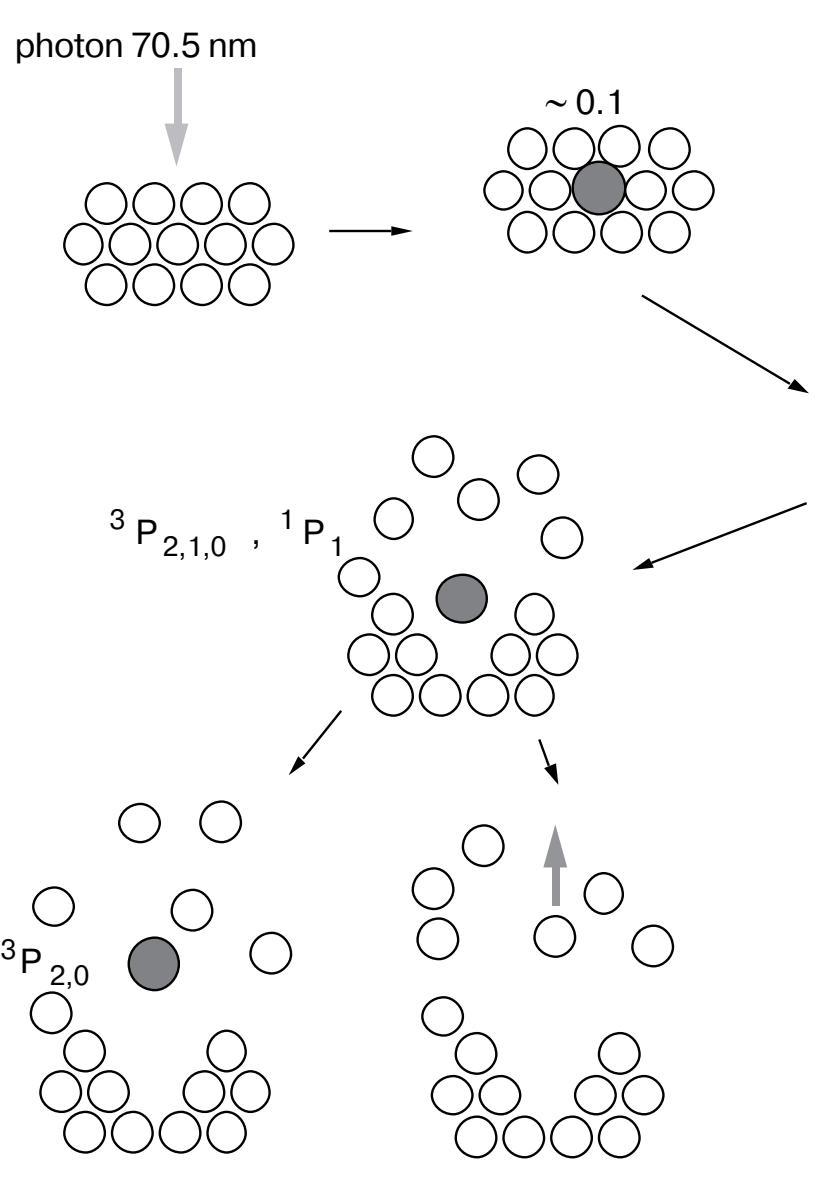

\section{B1 exciton}

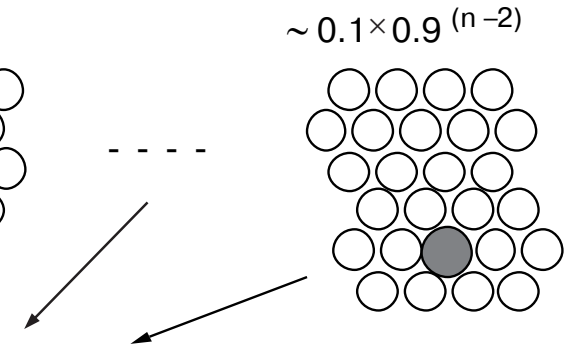

migration in solid

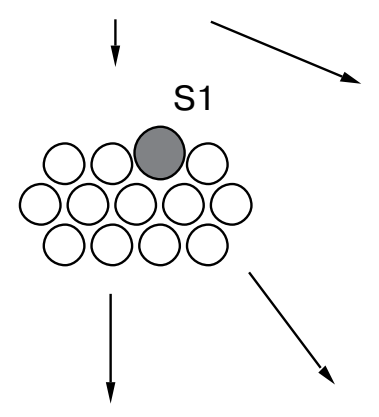

radiative decay
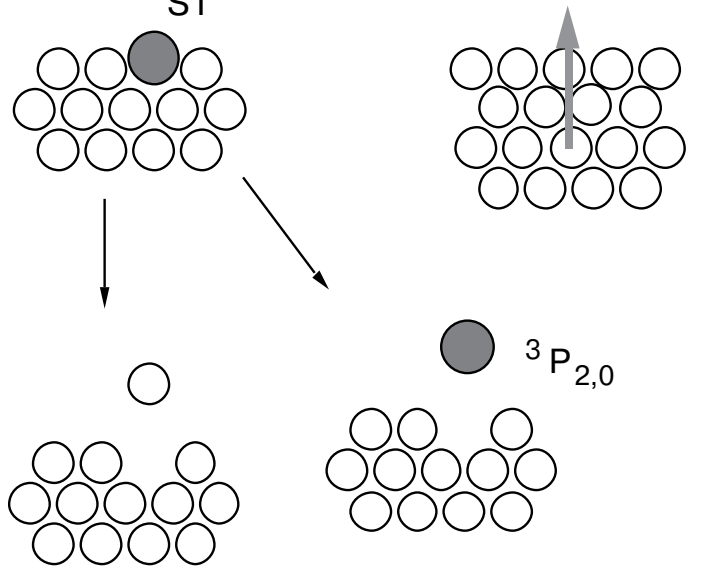

Fig. 5. Schematic chart of the relaxation cascade of the first order bulk exciton B1 in solid Ne.

tal number of desorption events is about $1 \%$ also in the case of $S^{\prime}$ excitation.

The relaxation cascade of the first order bulk exciton B1 of solid Ne are schematically shown in Fig. 5. When solid Ne is irradiated by $70.5 \mathrm{~nm}$ photons, the probability for the bulk exciton creation in the second underlying layer is estimated at 0.1 excitons / photon, 0.09 in the third layer, and $0.1(1-0.1)^{(n-2)}$ in the $n$th layer. Radiative decay may occur immediately or on the way of migration in the bulk. Some of them may reach the surface and convert into the surface exciton S1, which will follow the scenario in Fig. 4. The experimentally determined value of the metastable yield, $0.0014 \mathrm{Ne}^{*} /$ photon, via S1 state suggests that the total desorption yields via S1 excitons are about 0.2 atoms/photon considering the ratio of the total yield to the metastable one in the case of S1 excitation. This means that the conversion rate from $\mathrm{B} 1$ exciton to $\mathrm{S} 1$ exciton is 0.2 . The excited atom in the second or third layers can be squeezed out because of the large lattice distortion and can blow a number of atoms in the surface layer away. The desorption of metastable $\mathrm{Ne}$ from the underlying layers, which losses the kinetic energy by collisions with neutral atoms in the overlayers, was experimentally identified in the TOF spectra of Fig. 2 as a tail $D$. The large desorption yields of the order of unity brought by the bulk exciton can be attributed to this internal sputtering mechanism by excited atoms in the second and deeper layers beneath the surface [7].

The TOF spectrum of desorbed metastables by the excitation of B2 exciton is quite similar to that by $\mathrm{S}$ 'except the signal caused by a long life time (several hundreds $\mu \mathrm{s}$ ) luminescence. The relaxation of B2 into $\mathrm{S} 1, \mathrm{~S}^{\prime}$, and the excimer is clearly distinguished from the spectrum. Though the total number of excitons excited by B2 excitation is an essentially the same to that by $\mathrm{B} 1$ excitation, there is obvious difference between the total desorption yields by B1 and B2 excitations. This is probably because of the difference in the distribution of the initial excitation in the Ne film and because of the relaxation channel of B2, which yields the luminescence stated above.

\section{Desorption of $\mathrm{Kr}$}

\subsection{Metastable desorption}

The desorption of metastable species from solid Kr, observed by electron and high energy ion bombard- 
ment, is attributed to the ED mechanism. However, it has been reported that adsorption of a small amount of hydrogen makes it possible to desorb metastables by the $\mathrm{CE}$ mechanism by changing the electron affinity into negative [26]. Because of this phenomenon, it is difficult to determine the absolute desorption yields of a metastable via the process intrinsic of pure solid Kr. This is also the case for solid Xe.

\subsection{Total deSorption}

The wavelength dependence of the photo-desorption intensities from solid $\mathrm{Kr}$ for three different film thicknesses is shown in Fig. 6, where the curves are the direct output from the mass spectrometer with no compensation applied for a higher order light. The arrows show the wavelength corresponding to the excitation of the series of excitons in solid Kr. The coincidence between the exciton excitation energies and the peaks or the shoulders in the figure clearly shows that the excitons induce the desorption. The desorption yields were estimated by the peak height above the continuous background which was due to the bulk ionization caused by the higher order light from the monochromator. The desorption yield at S1 $(3 / 2)$ excitation has apparently no thickness dependence and is about 0.015 atoms/photon. The desorption by the bulk exciton creation becomes detectable at a few tens atomic layers in our experimental condition. The desorption yields at $\mathrm{B} 1(3 / 2)$ and B1 $(1 / 2)$ excitation increase with the film thickness and reach saturated values, 0.03 and 0.02 atoms / photon, respectively, at a thickness of about 100 atomic layers. The absolute yields of the total desorption by

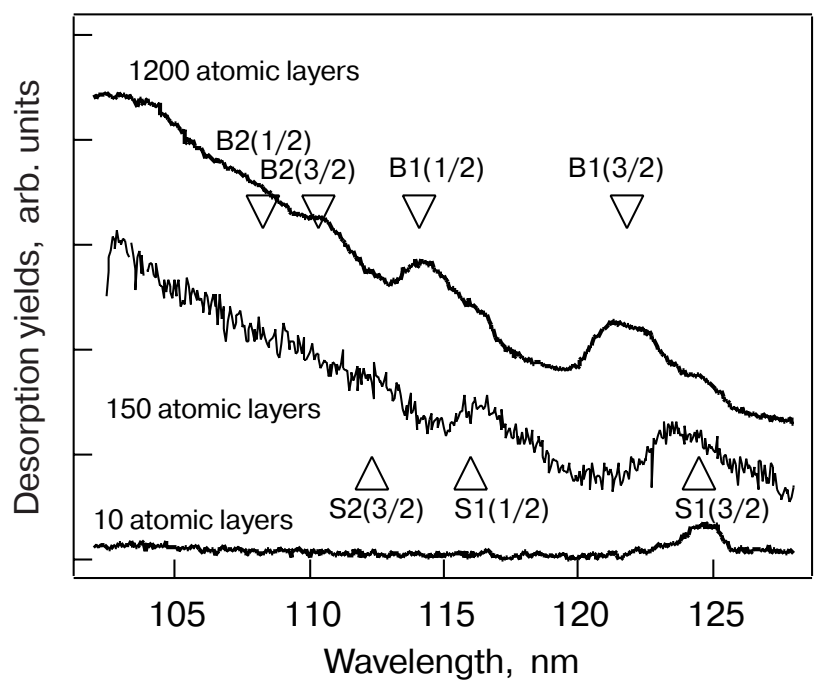

Fig. 6. the Total desorption yields as a function of incident wavelength for three different thicknesses of $\mathrm{Kr}$ films. the five different excitonic excitations are summarized in Table.

\subsection{DeSorption model and Simulation of total deSorption yieldS}

The desorption process caused by excimer dissociation and internal sputtering after the bulk exciton excitation is considered as sequential processes as schematically shown in Fig. 7: i) creation of a free exciton, ii) migration of the exciton, iii) formation of an excimer, iv) dissociation of an excimer, and v) collision cascade followed by internal sputtering. In order to calculate the desorption yields, each step should quantitatively be evaluated. The detail of the calculation will be published elsewhere [27]. Though the behavior of the exciton at a vacuum interface and metal substrate is not well understood, the photoemission data of $\mathrm{Kr}$ were well described by assuming that the metal surface is a perfect sink and that the vacuum interface is a perfect reflector for the exciton [28]. The final process $\mathrm{v}$ ), which is one of the most important factors to give the theoretical desorption yields, has extensively been studied by a classical molecular dynamic simulation [7]. A distribution of the initial excitation in the process i) and a diffusion length in the migration ii) affect the depth profile of the distribution of the trapped excimer and, therefore, determine the film thickness dependence of the total desorption yield. The initial distribution of the excited bulk excitons is estimated from the photo-absorption coefficient [29]. The only unknown parameter is the diffusion length $L$ of the free exciton, which is expected to be highly affected by crystal condition and temperature. As is shown in Fig. 8, both the absolute value and the thickness dependence were satisfactorily reproduced by choosing the parameter $L$ between 5 and $10 \mathrm{~nm}$. This value shows good agreement with the estimation between 1 and $10 \mathrm{~nm}$ by Schwentner et al.

i)

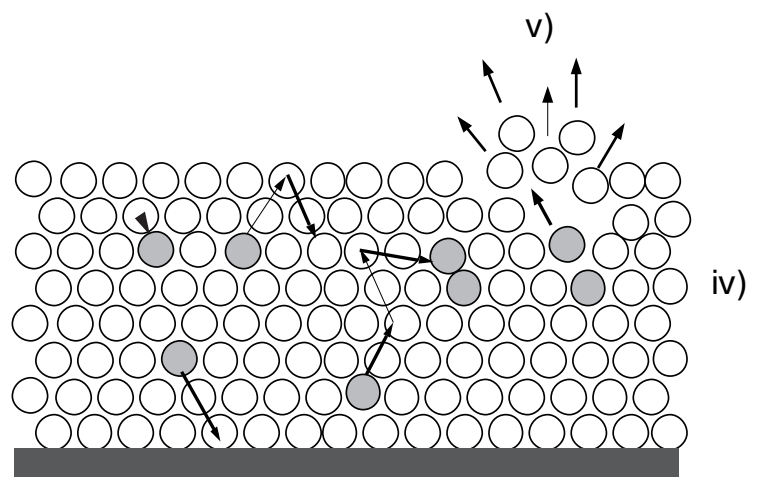

ii)

iii)

Fig. 7. The model of sequential steps of the excimer dissociation process in solid $\mathrm{Kr}$. 


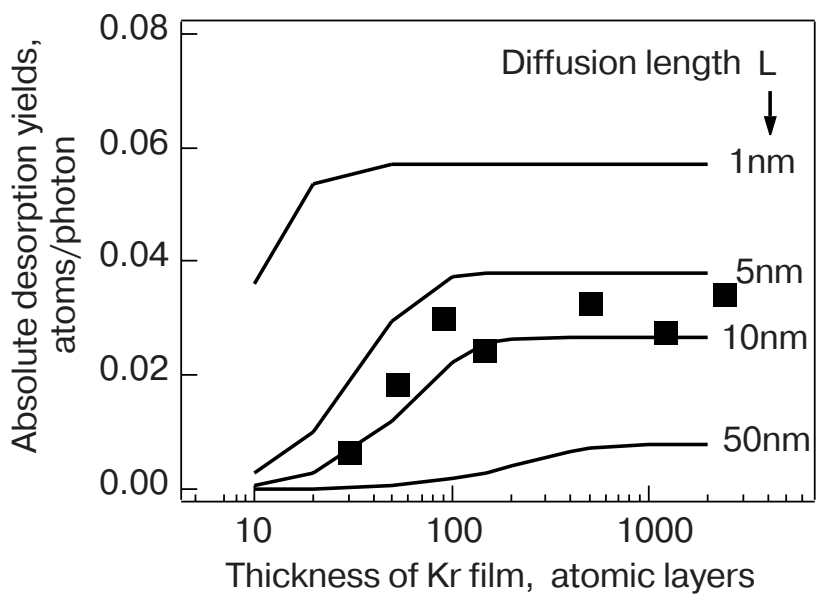

Fig. 8. Thickness dependence of the total desorption yield of solid $\mathrm{Kr}$; the experimental results (solid square) and the simulated ones (lines) for the diffusion length as a parameter.

from the photoemission study [28]. We have also applied this method of simulation to the ESD case of solid $\mathrm{Kr}$ and obtained satisfactory results [27].

Though we assume that the $\mathrm{CE}$ mechanism does not work at the surface of solid $\mathrm{Kr}$, the surface exciton excitation resulted in significant total desorption. This may be caused by the excimer dissociation on the surface. However, we cannot exclude the effect of hydrogen adsorption which makes the CE process effective.

\section{Desorption of Ar}

The desorption of metastables from solid Ar by both $\mathrm{CE}$ and ED mechanisms has been reported by many researchers $[24,30,31]$. There are some experimental difficulties, however, in determining the absolute yields of Ar metastable; unknown angular distribution of desorbed Ar, low signal intensity, and unstable detection efficiency of a secondary electron multipliers. Nevertheless we have obtained a rough estimation of the absolute metastable yield of $1 \cdot 10^{-5}$ atoms / photon by S1 excitation [12]. Though the determination of the absolute yields of total desorption has already been made in the same way as the $\mathrm{Kr}$ (see Table), the comparison between the experimental results and the simulation is not so straightforward as in the $\mathrm{Kr}$ case. It is expected for solid Ar that both $\mathrm{CE}$ and $\mathrm{ED}$ mechanisms contribute to the total desorption in comparable measure. The model calculation of the total yield at $\mathrm{B} 1$ excitation, in which only the ED mechanism is assumed under conditions similar to the $\mathrm{Kr}$ case, gives a theoretical desorption yield of almost one tenth of the experimental value. This large discrepancy is probably because the bulk excitons are efficiently converted to the surface ones at the vacuum interface and they are desorbed by the CE mechanism. The model simulation, which takes both CE and ED mechanisms into account is under progress.

\section{Summary}

We have measured the absolute yields of metastable and total desorption at the surface of solid $\mathrm{Ne}$, Ar, and $\mathrm{Kr}$ by the photostimulated desorption technique. The experimental results are well explained by the model in which the excitons play a crucial role. However the mechanism which leads to the desorption for each rare gas is different. For solid Ne, the predominant mechanism which works for both metastable and total desorptions is the $\mathrm{CE}$ one. The large lattice distortion energy makes the internal sputtering process possible and results in a large total desorption yield of the order of 1 atoms / photon by bulk exciton excitation. For solid $\mathrm{Kr}$, the desorption process can be well described solely with the ED mechanism in which the migration of a free exciton before trapping into the excimer state determines the absolute yields and its film thickness dependence. In solid Ar, both CE and ED mechanisms work for the desorption, which make the model calculation of the desorption yield difficult. The mechanism which works in the desorption process from solid Xe must be solely the ED one as is the case for solid Kr. Unfortunately, we have not obtained the data on absolute total desorption yields which can be compared with the simulation result. This is because of the experimental difficulty due to the low desorption signal intensity and the limitation of our experimental apparatus.

It was demonstrated that the desorption study could reveal the dynamics of the excitons in rare gas solids. The promising step in the future study may be a systematic investigation of the temperature dependence of the desorption phenomena on a well defined sample crystal. The desorption study at the surface of a rare gas alloy [32] will also yield interesting information on the dynamics of the exciton in a heterogeneous system.

\section{Acknowledgment}

This work was supported by the Joint Studies Program of the Institute for Molecular Science and partly by a Grant-in-Aid for Scientific Research from the Ministry of Education, Science, Sports, and Culture, Japan. 
1. I. Arakawa, Mol. Cryst. Liq. Cryst. 314, 47 (1998).

2. F. Colletti, J.M. Debever, and G. Zimmerer, J. Phys. Lett. 45, 467 (1984).

3. P. Feulner, T. Muller, A. Puschmann, and D. Menzel, Phys. Rev. Lett. 59, 791 (1987).

4. T. Kloiber and G. Zimmerer, Radiat. Eff. Def. Sol. 109, 219 (1989).

5. N. Scwentner, F.-J. Himpsel, V. Saile, M. Skibowski, W. Steinmann, and E.E. Koch, Phys. Rev. Lett. 34, 528 (1975).

6. D.E. Weibel, A. Hoshino, T. Hirayama, M. Sakurai, and I. Arakawa, in: Desorption Induced by Electronic Transitions DIET V, E.B. Stechel, A. Burns, and D.R. Jennison (eds.), Springer, Berlin (1993), p. 333.

7. S. Cui, R.E. Johnson, and P.T. Cummings, Phys. Rev. B39, 9580 (1989).

8. N.E. Small-Warren and L.-Y. Chow Chiu, Phys. Rev. A11, 1777 (1975).

9. T.R. Connor and M.A. Biondi, Phys. Rev. A140, 778 (1965).

10. I.Ya. Fugol', O.N. Grigorashchenko, A.N. Ogurtsov, and E.V. Savchenko, J. Luminescence 53, 517 (1992).

11. M. Sakurai, T. Hirayama, and I. Arakawa, Vacuum 41, 217 (1990)

12. T. Hirayama, A. Hayama, T. Koike, T. Kuninobu, I. Arakawa, K. Mitsuke, M. Sakurai, and E.V. Savchenko, Surf. Sci. 390, 266 (1997).

13. I. Arakawa, T. Adachi, T. Hirayama, and M. Sakurai, Surf. Sci. 451, 136 (2000).

14. J.A.R. Samson, J. Opt. Soc. Am. 54, 6 (1964).

15. I. Arakawa, D.E. Weibel, T. Nagai, M. Abo, T. Hirayama, M. Kanno, K. Mitsuke, and M. Sakurai, Nucl. Instrum. Meth. Phys. Res. B101, 195 (1995).

16. M. Sakurai, T. Nagai, M. Abo, T. Hirayama, and I. Arakawa, J. Vac. Soc. Jpn. 38, 298 (1995).
17. D.E. Weibel, T. Hirayama, and I. Arakawa, Surf. Sci. 283, 204 (1993).

18. M. Sakurai, T. Adachi, T. Hirayama, and I. Arakawa, UVSOR Activity Report 2001, UVSOR-29, 58 (2002).

19. T. Kuninobu, A. Hayama, T. Hirayama, and I. Arakawa, Surf. Sci. 390, 272 (1997).

20. A. Hayama, T. Kuninobu, T. Hirayama, and I. Arakawa, J. Vac. Sci. Technol. A16, 979 (1998).

21. V. Saile and E. E. Koch, Phys. Rev. B20, 784 (1979).

22. K. Inoue, H. Sakamoto, and H. Kanzaki, Solid State Commun. 49, 191 (1984).

23. T. Hirayama, T. Nagai, M. Abo, I. Arakawa, K. Mitsuke, and M. Sakurai, J. Electr. Spectr. Rel. Phen. 80, 101 (1996).

24. I. Arakawa and M. Sakurai, in DeSorption Induced by Electronic Transitions DIET IV, G. Betz and P. Varga (eds.), Springer, Berlin (1990), p. 246.

25. D. Pudewill, F. Himpsel, V. Saile, N. Schwentner, M. Skibowski, and E.E. Koch, Phys. Status Solidi. B74 485 (1976).

26. Hayama, T. Kuninobu, T. Hirayama, and I. Arakawa, J. Vac. Sci. Technol. A16, 979 (1998).

27. T. Adachi, T. Hirayama, T. Miura, I. Arakawa, and M. Sakurai, Surf. Sci. (to be published).

28. N. Schwentner, G. Martens, and H.W. Rudolf, Phys. Status Solidi B106, 183 (1981).

29. B. Sonntag, in Rare Gas solids, vol. II, M.L. Klein and J.A. Venables (eds.), Academic, New York (1977).

30. D.J. O'Shaughnessy, J.W. Boring, S. Cui, and R.E. Johnson, Phys. Rev. Lett. 61, 1635 (1988).

31. I. Arakawa, M. Takahashi, and K. Takeuchi, J. Vac. Sci. Technol. A7, 2090 (1989).

32. D.E. Weibel, T. Nagai, T. Hirayama, I. Arakawa, and M. Sakurai, Langmuir 12, 193 (1996). 